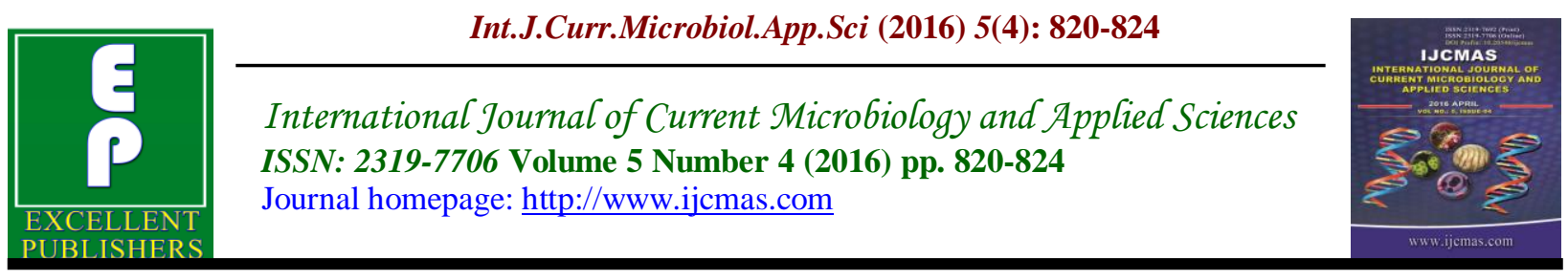

Original Research Article

http://dx.doi.org/10.20546/ijcmas.2016.504.094

\title{
Speciation and Antifungal Susceptibility Pattern of Candida Isolated from Clinical Specimens
}

\author{
S. Mohan* and D. Karthikeyan \\ Department of Microbiology, Vinayaka Missions Medical College and Hospital, \\ Karaikal-609609 Puducherry, India \\ *Corresponding author
}

\begin{abstract}
A B S T R A C T
Keywords

Candida, CHROM agar, Antifungal susceptibility.

\section{Article Info}

Accepted:

24 March 2016

Available Online:

10 April 2016

Candidiasis is one of the major fungal infections among hospitalized and immunocompromised patients. Identification of Candida upto species level is required as there is increase in the incidence of non albican Candida infections. Increased incidence of antifungal resistance has also been reported. Hence, this study was aimed at speciation of candida and their antifungal susceptibility from clinical specimens. A total of 93 Candida isolated from various clinical specimens. Candida Speciation was performed using CHROM agar and conventional methods. Antifungal susceptibility test was performed as per CLSI recommendations in document M44-A. Candida albicans (39.78\%) was found to be the predominant species isolated from clinical specimens followed by non albican candida. Among non albican candida, C.tropicalis $(22.58 \%)$ was predominant species isolated. C.albicans $(45.95 \%)$ and C.tropicalis $(38.09 \%)$ showed least sensitivity towards azole group. Majority of isolates remained susceptible to Amphotericin B. Candida albicans was found to be the predominant species isolated from clinical specimens. Among non albicans candida, Candida tropicalis was the predominant species isolated. Candida tropicalis was least sensitive to azoles followed by Candida albicans.
\end{abstract}

\section{Introduction}

Candida species are the members of the normal flora of the skin, mucous membranes, and gastrointestinal tract. Candidiasis is a common fungal disease found in humans affecting, mucosa, skin, nails and internal organs of the body. Candida albicans is by far the most common species causing infections in humans. (Fridkin, 2006) However, non albicans Candida species are also being implicated in recent years (Gullo, 2009).
Candida is the sixth most common isolated nosocomial pathogen, especially from urinary tract.It is the fourth most common cause of blood stream infection. (Fridkin, 1996) Predisposing factors for candida infections are: prolonged use of antimicrobial agents, immunocompromised status, steroids, chemotherapy, and catheterization (Manchanda, 2011).

Species level identification of Candida is important for the treatment, as not all 
species respond to the same treatment because of the problem of anti-fungal resistance. (Shivanand, 2011) Azole group of drugs, are commonly used in treating many forms of Candidal infections for a long time, however, their prolonged use has led to the development of drug resistance in C.albicans and other species. (Sachin, 2012) Amphotericin B, a polyene fungicidal agent, has been used for the treatment for invasive Candidal infections, but cost and dose related side effects limit its use. (Marchetti, 2003)

Hence, this study was aimed at speciation of candida and their antifungal susceptibility from clinical specimens.

\section{Materials and Methods}

A total of 93 Candida isolated from various clinical specimens submitted to the microbiology laboratory from different out patients and in patients departments of VM M C \& H Karaikal, were included in the study. All suspected yeast colonies on sheep blood agar were confirmed by Gram staining. All such yeast isolates were sub cultured on chromogenic medium, HiMedia $\mathrm{CHROM}$ agar and incubated at $37^{\circ} \mathrm{C}$ for 24 hours and the species were identified by type and colour of the colonies on CHROM agar media as per manufacturer's instructions.

C.albicans - blue green

C.tropicalis - dark blue gray centre with pink halo

C. krusei - pink large rough spreading colonies with pale edge.

C.parapsilisis - pale cream coloured colonies.

C.dubliniensis - dark green colonies.
All isolates were further inoculated on corn meal agar (CMA) by slide culture method to determine microscopic morphological features of various Candida species. (Marchetti, 2011)

Antifungal susceptibility test was performed as per CLSI recommendations in document M44-A. (Wayne Pa, 2004) Muller Hinton Agar supplemented with glucose and methylene blue, was prepared (Lee, 2001; Pfaller, 2004) Following antifungal discs were used amphotericin B (100 units), fluconazole $(10 \mathrm{mcg})$, clotrimazole $(10 \mathrm{mcg})$, itraconazole $(10 \mathrm{mcg})$, ketaconanole (10 $\mathrm{mcg})$.

\section{Quality control strain:}

\section{C.albicans (ATCC90028)}

Statistical analysis was done by simple percentage method.

\section{Results and Discussion}

A total of 93 Candida spp. were isolated from various clinical specimens. Candida albicans (39.78\%) was found to be the predominant species isolated from clinical specimens followed by non albican candida. Among non albican candida, C.tropicalis $(22.58 \%)$ was predominant species isolated (Table.1) C.albicans (45.95\%) and C.tropicalis (38.09\%) showed least sensitivity towards azole group. Majority of isolates remained susceptible to Amphotericin B.(Table.2)

The pathogenesis of candida infections are extremely complex and probably varies with each species. Though over 100 species of candida have been recognized, only few have been found to cause human infections. (Krause, 2005) Candida albicans is generally considered as the major pathogen among the Candida species. 
Table.1 Distribution of Candida Species among Various Clinical Specimems

\begin{tabular}{|l|l|l|l|l|l|}
\hline $\begin{array}{l}\text { Clinical } \\
\text { specimen }\end{array}$ & C.albicans & C.tropicalis & C.parapsilosis & C.krusei & C.dubiliniensis \\
\hline urine & $12(32.43 \%)$ & $7(33.33)$ & $4(23.53 \%)$ & $4(30.77 \%)$ & - \\
\hline $\begin{array}{l}\text { Respiratory } \\
\text { specimens }\end{array}$ & $15(40.55 \%)$ & $9(42.86 \%)$ & $4(23.53 \%)$ & - & - \\
\hline $\begin{array}{l}\text { Pus/pus } \\
\text { swabs }\end{array}$ & $2(5.41 \%)$ & $1(4.76 \%)$ & - & $2(15.38 \%)$ & - \\
\hline $\begin{array}{l}\text { Vaginal } \\
\text { swabs }\end{array}$ & $5(13.51 \%)$ & $3(14.29 \%)$ & $1(5.88 \%)$ & $1(7.70 \%)$ & $1(20 \%)$ \\
\hline CSOM & $1(2.70 \%)$ & $1(4.76 \%)$ & - & $1(7.70 \%)$ & - \\
\hline Blood & $1(2.70 \%)$ & - & $3(17.65 \%)$ & $5(38.45 \%)$ & $1(20 \%)$ \\
\hline Stool & $1(2.70 \%)$ & - & $5(29.41 \%)$ & - & $3(60 \%)$ \\
\hline Total & $37(100 \%)$ & $21(100 \%)$ & $17(100 \%)$ & $13(100 \%)$ & $5(100 \%)$ \\
\hline
\end{tabular}

Table.2 Antifungal Susceptibility Pattern of Candida Isolates

\begin{tabular}{|l|l|l|}
\hline Candida species & $\begin{array}{l}\text { Sensitive against Azole } \\
\text { group }\end{array}$ & Sensitive against Amphotericin B \\
\hline C.albicans (37) & $17(45.95 \%)$ & $35(94.59 \%)$ \\
\hline C.tropicalis (21) & $8(38.09 \%)$ & $20(95.24 \%)$ \\
\hline C.parapsilosis (17) & $14(82.35 \%)$ & $17(100 \%)$ \\
\hline C.krusei (13) & $11(84.61 \%)$ & $13(100 \%)$ \\
\hline C.dubiliniensis (5) & $3(60 \%)$ & $5(100 \%)$ \\
\hline
\end{tabular}

An increase in the prevalence of nonalbicans species has been noted during the last decades. The potential clinical importance of species-level identification has been recognized as Candida species differ in the expression of virulence factors and antifungal susceptibility. In the present study C.albicans (39.78\%) was the predominant isolate. In our study the incidence of non albicans candida was $60.2 \%$.Various studies stated that the incidence of non albicans candida ranges from 54-75\%. (Golia, 2013) A study by Vijaya et al. (Vijaya, 2011) showed nonalbicans candida $(54.1 \%)$ to have a higher incidence than C.albicans (45.9\%). A study by Manchanda et al. showed non- albicans candida $(72.4 \%)$ to have a higher incidence than C.albicans (27.5\%). Among the non albicans species, Candida tropicalis $(37.5 \%)$ was the predominant isolate followed by C.parapsilosis (30.3\%). C. tropicalis is the most virulent of the non- albicans candida, this may be due to its ability to adhere to epithelial cells and its ability to secrete moderate levels of proteinases. (Moran, 2002)

Candida species were initially susceptible to 'Azoles', but now several species have developed resistance to the azoles. Widespread use of fluconazole for the prophylaxis and treatment of candidiasis has led to a reduction in the number of cases of infections caused by Candida albicans but has also resulted in the emergence of candidal infections caused by fluconazoleresistant Candida non albicans. (Gupta, 2001) In our study, candida tropicalis was least sensitive to azoles $(38.09 \%)$ followed 
by Candida albicans (48.95\%).Patel et al have reported that Azole group showed $25.5 \%$ sensitive among C.albicans and $18.7 \%$ sensitive among $C$. tropicalis to fluconazole while in Amphotericin B, sensitivity varied from $75.6 \%$ to $100 \%$ to all isolated species of candida. (Patel, 2012) In our study, two strains of Candida albicans and one strain of Candida tropicalis showed resistance to amphotericin B. In another study by Deorukhkar et al. (Deorukhkar, 2013). It was seen that the resistant rate of Candida to fluconazole and amphotericn B was $27.3 \%$ and $5.8 \%$ respectively. The limitation in this study was that no other method was used to confirm the identity of the Candida isolates like the conventional carbohydrate fermentation and assimilation tests.

In conclusion, Non albicans Candida species are increasingly being isolated from clinical specimens. Among non albicans candida, Candida tropicalis was the predominant species isolated. Candida tropicalis was least sensitive to azoles followed by Candida albicans. Hence speciation and Susceptibility testing of the candida isolates plays an important role in the management of candidal infections. CHROM agar is a simple, rapid and inexpensive method for the identification of such candida species.

\section{References}

Agarwal, S., Manchanda, V., Verma, N., Bhalla, P. 2011. Yeast identification in routine clinical microbiology laboratory and its clinical relevance. Ind. J. Med. Microbiol., 29(2): 172177.

Deorukhkar, S.C., Saini, S., Mathew, S. 2014. Virulence factors contributing to pathogenicity of Candida tropicalis and its antifungal susceptibility profile. Int. J.

\section{Microbiol.}

Fridkin, S.K., Jarvis, W.R. 1996. Epidemiology of Nosocomial fungal infection. Clin. Microbiol. Rev., 9: 499-511.

Fridkin, S.K., Kaufman, D., Edwards, J.R., Shetty, S., Horan, T. 2006. Changing incidence of Candida Bloodstream infections among NICU patients in United States: 1995-2004. Pediatr., 117: $1680-87$.

Golia, S., Reddy, K.M., Karjigi, K.S., Hittinahalli, V. 2013. Speciation of Candida using chromogenic and cornmeal agar with determination of fluconazole sensitivity. Al Ameen J. Med. Sci., 6(2): 163-166.

Moran, G.P., Sullivan, D.J., Coleman, D.C. 2002. Emergence of non-Candida albicans Candida species as pathogens. In: Calderone RA. Candida and Candidiasis. 4th Edition, 4: 37-53.

Gullo, A. 2009. Invasive fungal infections: the challenge continues. Drugs, 69: 65-73.

Gupta, N., Mittal, N., Sood, P., Kumar, S., Kaur, R., Mathur, M.D. 2001. Candidemia in neonatal Intensive Care Unit. Indian J. Pathol. Microbiol., 44(1): 45-8.

Krause, R., Reisinger, E. 2005. Candida and antibiotic associated diarrhea. Clin. Microbiol. Infect., 11: 1-2.

Lee, S.C., Fung, C.P., Lee, N., See, L.C., Huang, J.S., Tsai, C.J., Chen, K.S., Shieh, W.B. 2001. Fluconazole Disk Diffusion Test with Methylene Blueand Glucose-Enriched MuellerHinton Agar for Determining Susceptibility of Candida Species, $J$. Clin. Microbiol., 39(4): 1615-1617.

Marchetti, O., Moreillon, P., Entenza, J.M., Vouillamoz, J., Glauser, M.P., Bille, J., et al. 2003. Fungicidal synergism of FLU and cyclosporine in Candida 
albicans is not dependent on multidrug efflux transporters encoded by CDR1, CDR2, CaMDR1, and FLU1 genes. Antimicrob. Agents Chemother., 47: 1565-70.

Patel, L.R., Pethani, J.D., Bhatia, P., Rathod, S.D., Shah, P.D. 2012. Prevalence of Candida Infection and its Antifungal Susceptibility Pattern in Tertiary Care Hospital, Ahmedabad. Natl. J. Med. Res., 2(4): 439-441.

Pfaller, M.A., Boyken, L., Messer, S.A., Hollis, R.J., Diekema, D.J. 2004. Stability of Mueller-Hinton Agar Supplemented with Glucose and Methylene Blue for Disk Diffusion Testing of Fluconazole and Voriconazole. J. Clin. Microbiol., 42(3): 1288-1289.

Sachin, C., Deorukhkar, Dr. Santosh, Saini. 2012. Species distribution and antifungal susceptibility profile of
Candida species isolated from blood stream infections. J. Evol. Med. Dent. Sci., 1(3): 241-249.

Shivanand Dharwad, Saldanha Dominic, R.M. 2011. Species identification of candida isolates in various clinical specimens with their anti-fungal susceptibility patterns. J. Clin. Diag. Res., 5(6): 1177-1181.

Manchanda, V., Agarwal, N., Verma. 2011. Yeast identification in routine clinical Microbiology laboratory and its clinical relevance. Indian J. Med. Microbiol., 29(2): 172.

Vijaya, D., Harsha, T.R., Nagaratnamma, T. 2011. Candida speciation using chrom agar. J. Clin. Diag. Res., 5(4): 75-57.

Wayne, Pa. 2004. National committee for antifungal disk diffusion susceptibility testing yeast: approved guidelines, $C L S I$, M-44A.

\section{How to cite this article:}

Mohan, S., and Karthikeyan, D. 2016. Speciation and Antifungal Susceptibility Pattern of Candida Isolated from Clinical Specimens. Int.J.Curr.Microbiol.App.Sci.5(4): 820-824. doi: http://dx.doi.org/10.20546/ijcmas.2016.504.094 